\title{
Analog VLSI Neuromorphic Network with Programmable Membrane Channel Kinetics
}

\author{
Theodore $\mathrm{Yu}^{1,2,4}$ and Gert Cauwenberghs $s^{2,3,4}$ \\ ${ }^{1}$ Electrical and Computer Engineering Department, Jacobs School of Engineering \\ ${ }^{2}$ Neurobiology Section, Division of Biological Sciences \\ ${ }^{3}$ Bioengineering Department, Jacobs School of Engineering \\ ${ }^{4}$ Institute for Neural Computation \\ University of California San Diego, La Jolla, CA 92093
}

\begin{abstract}
We demonstrate neuron spiking dynamics in a small network of analog silicon neurons with dynamical conductancebased synapses. The analog VLSI chip (NeuroDyn) emulates analog continuous-time dynamics in a fully digitally programmable network of 4 biophysical neurons. Each neuron in NeuroDyn implements Hodgkin-Huxley dynamics in 4 variables, with 28 parameters defining the conductances, reversal potentials, and voltage-dependence of the channel kinetics. All 12 chemical synapses interconnecting the neurons also have individually programmable parameters defining conductance, reversal potential, and pre/post-synaptic voltage dependence of the channel kinetics. All configurable parameters in the implemented model have a biophysical origin, thus supporting direct interpretation of the results of adapting/tuning the parameters in terms of neurobiology. Uniform temporal scaling of the dynamics of membrane and gating variables is demonstrated by tuning a single current parameter, yielding variable speed output exceeding real time. The $0.5 \mu \mathrm{m}$ CMOS chip measures $3 \mathrm{~mm} \times 3 \mathrm{~mm}$, and consumes $1.29 \mathrm{~mW}$.
\end{abstract}

\section{INTRODUCTION}

Neuromorphic engineering [1] takes inspiration from neurobiology in the design of artificial neural systems in silicon integrated circuits, based upon function and structural organization of biological nervous systems. Investigation of neural behavior on large scale requires modeling and implementation of individual neurons and their synaptic connections. Hodgkin and Huxley's seminal work in the investigation and formalization of neuron dynamics have long been the standard of biophysical accuracy [2]. However, the primary drawback of the Hodgkin and Huxley formalism is the difficulty of implementing the vast number of parameters and the resulting complexity. Previous hardware implementations of neurons and synapses are typically limited to static modeling in an integrate-andfire framework. Though this approach is generally convenient and computationally efficient, passive modeling of the currents not only lacks insight from biological structure, but also lacks accuracy in modeling the timing of the postsynaptic action potentials. Several integrated circuit implementations of Hodgkin-Huxley neurons have been formulated and demonstrated [3], [4], [5], [6], [7] and [8]. Here we present a network of Hodgkin-Huxley neurons and conductance-based synapses that accurately models the detailed rate-based kinetics of membrane channels in the neural and synaptic dynamics.
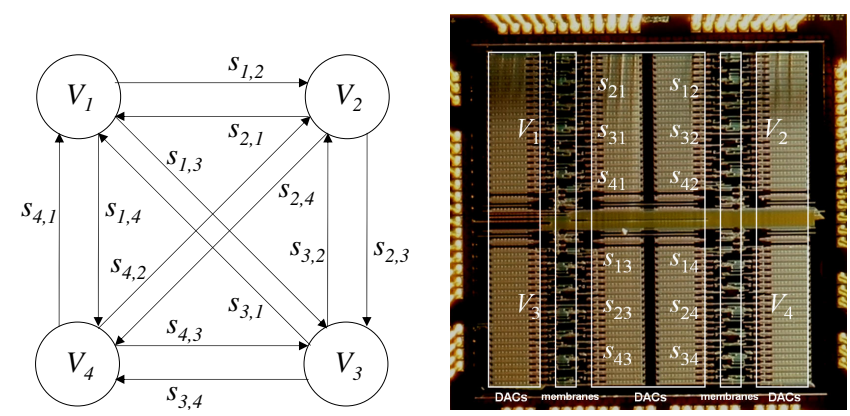

Fig. 1. NeuroDyn system diagram (a), and chip micrograph (b). Four neurons are interconnected by twelve synapses, each with programmable channel kinetics, conductances, and reversal potentials (see Table 1).

The voltage dependence of the channel opening and closing rates is approximated through programmable analog regression functions.

\section{A. NeuroDyn Overview}

The NeuroDyn Board consists of 4 Hodgkin-Huxley based neurons fully connected through 12 conductance-based synapses as shown in Fig. 1(a). All parameters are individually addressable and individually programmable and are biophysically-based governing the conductances, reversal potentials, and voltage-dependance of the channel kinetics. There are a total of 384 programmable parameters governing the dynamics as shown in Table I. Each parameter is stored onchip in a 10-bit DAC.

\section{B. Chip Architecture}

The NeuroDyn chip is organized into four quadrants with each quadrant containing one neuron, its associated membrane capacitance and the three synaptic inputs from the other neurons. Notice that each channel current and synaptic current follow the same general form as illustrated in Fig. 2. The channel current is a product of a conductance term modulated by a product of gating variables and the difference between the membrane voltage and reverse potential as illustrated below in (1) and (2). The similar form for both the neuron channel currents and synaptic current allow for a small number of 
TABLE I

NEURoDyn DAC PARAMETERS

$\begin{array}{rccc}\text { Neurons } \mathrm{V}_{i}: & & & \\ \alpha_{n_{i}}(V) & \beta_{n_{i}}(V) & \mathrm{g}_{N a_{i}} & \mathrm{E}_{N a_{i}} \\ \alpha_{m_{i}}(V) & \beta_{m_{i}}(V) & \mathrm{g}_{K_{i}} & \mathrm{E}_{K_{i}} \\ \alpha_{h_{i}}(V) & \beta_{h_{i}}(V) & \mathrm{g}_{L_{i}} & \mathrm{E}_{L_{i}} \\ 4 \times 3 \times 7^{*} & 4 \times 3 \times 7^{*} & 4 \times 3 & 4 \times 3 \\ \text { Synapses } \mathrm{s}_{i j}: & & & \\ \alpha_{r_{i j}}\left(V_{\text {pre }}\right) & \beta_{r_{i j}}\left(V_{\text {post }}\right) & \mathrm{g}_{s y n_{i j}} & \mathrm{E}_{s y n_{i j}} \\ 12 \times 7^{*} & 12 \times 7^{*} & 12 & 12\end{array}$

*All rates $\alpha, \beta$ are functions of voltage as 7-point sigmoidal spline.

circuits to model each component of the channel current. These circuits are explained in further detail below.

\section{BIOPHYSICAL MODELS}

\section{A. Membrane Dynamics}

The Hodgkin-Huxley membrane dynamics including conductance-based synapses is described by

$$
C_{m e m} \frac{d V_{i}}{d t}=-I_{N a_{i}}-I_{K_{i}}-I_{L_{i}}-\sum_{j} I_{s y n_{i j}}
$$

where $i, j=0 \ldots 3$, and

$$
\begin{aligned}
I_{N a_{i}} & =g_{N a_{i}} m_{i}{ }^{3} h_{i}\left(V_{i}-E_{N a_{i}}\right) \\
I_{K_{i}} & =g_{K_{i}} n_{i}{ }^{4}\left(V_{i}-E_{K_{i}}\right) \\
I_{L_{i}} & =g_{L_{i}}\left(V_{i}-E_{L_{i}}\right) \\
I_{s y n_{i j}} & =g_{s y n_{i j}} r_{i j}\left(V_{i}-E_{s y n_{i j}}\right)
\end{aligned}
$$

\section{B. Channel Kinetics}

The gating variables $n_{i}, m_{i}$, and $h_{i}$ in the HH neuron model, as well as the rate variables $r_{i j}$ in the synapse dynamics, are modeled by a rate-based approximation to the kinetics governing the random opening and closing of membrane channels [9]:

$$
\frac{d n}{d t}=\alpha(1-n)-\beta n
$$

where $\alpha$ and $\beta$ are the voltage dependent opening and closing rates of the channel gates, and $n$ (which stands for any of the $n, m, h$, and $r$ variables) is the fraction of channel gates in the open state.

\section{NEUROMORPHIC IMPLEMENTATION}

The membrane dynamics and general rate-based models of voltage-gated channel kinetics are emulated in silicon. We model the voltage dependence of each of the opening and closing rates as a 7-point sigmoidal spline regression function. The voltage bases of the splines are linearly spaced, and the amplitudes are individually programmable (including sign selection), allowing for large and varied model space.

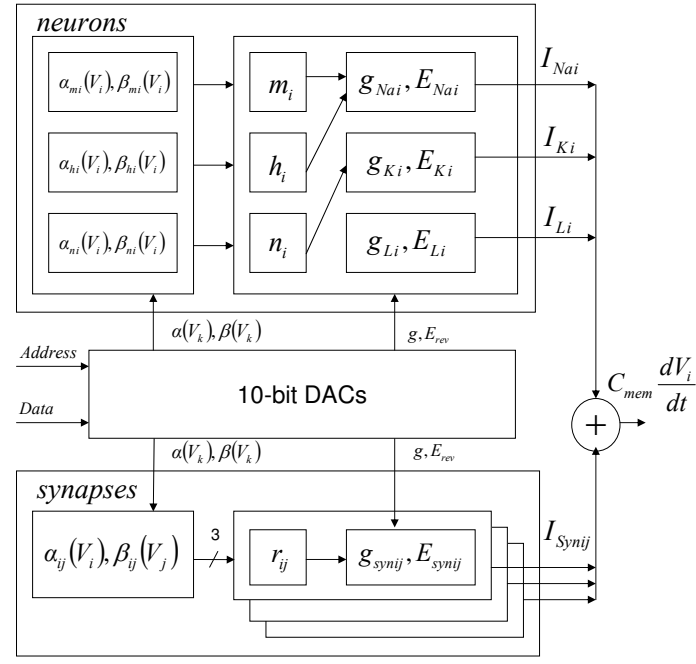

Fig. 2. System diagram for one of the four neurons in the NeuroDyn chip.

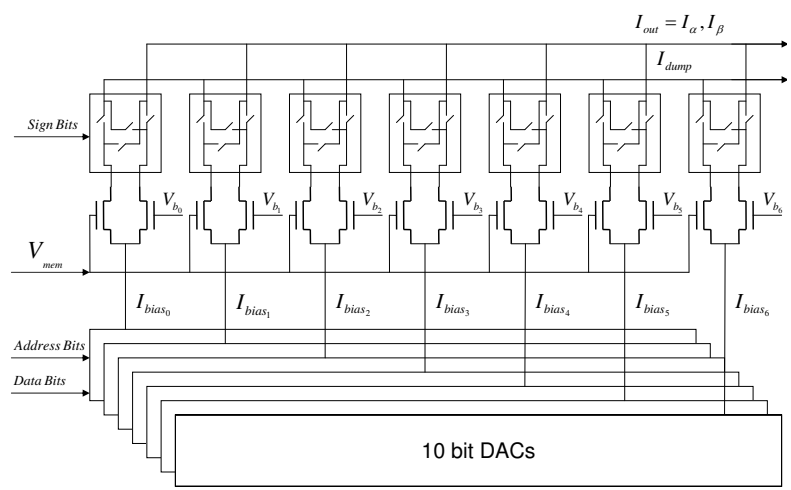

Fig. 3. Sigmoidal regression of voltage dependence of channel rate variables $\alpha$ and $\beta$.

\section{A. Voltage Dependent Channel Kinetics}

Opening rates $\alpha$ and closing rates $\beta$ are modeled with 7 point sigmoidal regression functions as illustrated in Fig. 3 . Each sigmoid is implemented by a simple differential pair. The bias current for the differential pair determines the height of the sigmoid while the bias voltage determines the location of the sigmoid. An additional switch circuit controlled by a sign bit determines the slope of the output current. By properly setting the voltage bias, current bias, and sign bit for each of the 7 sigmoidal functions, the summation can accommodate a wide range of functions approximating typical rate functions $\alpha$ and $\beta$ in the current domain $\left(I_{\text {out }}=I_{\alpha}\right.$ or $\left.I_{\beta}\right)$.

$$
I_{\text {out }}=\sum_{k=1}^{7} I_{\text {sig }, k}=\sum_{k=1}^{7} \frac{I_{\text {bias }, k}}{1+e^{ \pm \kappa\left(V_{\text {bias }, k}-V_{m}\right) / U_{t}}}
$$

Gating variables $m_{i}, h_{i}, n_{i}$, and $r_{i j}$ are implemented by the log-domain circuit shown in Fig. 4, which implements the kinetics (3) as:

$$
C V_{T} \frac{d}{d t} \frac{I_{\text {out }}}{I_{\text {ref }}}=I_{\alpha}\left(1-\frac{I_{\text {out }}}{I_{\text {ref }}}\right)-I_{\beta} \frac{I_{\text {out }}}{I_{\text {ref }}}
$$




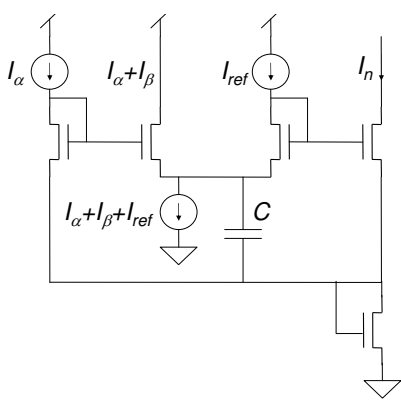

Fig. 4. Log-domain circuit implementing channel kinetics $\frac{d n}{d t}=\alpha(1-n)-$ $\beta n$.

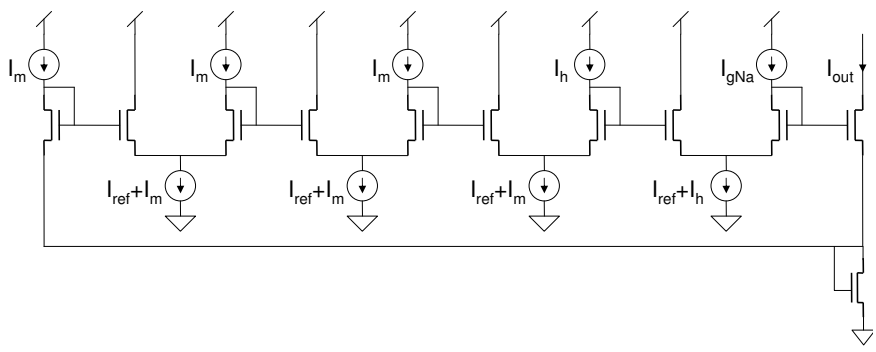

Fig. 5. Translinear circuit implementing gated conductances of the form $g x^{3} y$ such as $g_{N a} m^{3} h$ and $g_{K} n^{4}$. Synaptic gated conductances $g_{s y n} r$ are implemented by a 2 -stage version of the 5 -stage translinear circuit shown.

where $V_{T}=k T / q$ is the thermal voltage, and $I_{r e f}$ is a current reference that only affects the amplitude scale of the gating variables, but not the temporal scale of their dynamics. A previous implementation by Hynna et al. in [10] uses just a few transistors to approximate the rate-based kinetics (3), but suffers from the effects of the transistor backgate effect. Additional transistors in the circuit of [10] implement saturation in the opening and closing rates necessary for realistic time scales in the channel kinetics. Our circuit offers 14 parameters specifying the detailed voltage dependence of the opening and closing rates offering more flexibility in accurately modeling the channel kinetics.

\section{B. Gated conductances}

A translinear multiplier shown in Fig. 5 implements gating of the membrane conductances with the gating variables. A translinear multiplier exploits the zero sum of voltages along a loop to implement a multiplication of current sources [3]:

$$
I_{g_{N a} m^{3} h}=I_{g}\left(\frac{I_{m}}{I_{\text {ref }}}\right)^{3} \frac{I_{h}}{I_{r e f}}
$$

with similar constructs to implement the other gated conductances.

\section{Membrane dynamics}

Each membrane conductance is implemented by a differential transconductance amplifier, linearized through shunting in the differential pairs for wide dynamic range in subthreshold MOS operation [11]. Unity gain connection of the amplifier

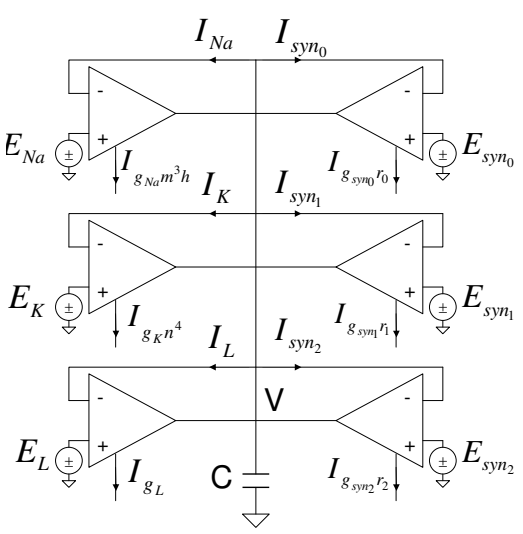

Fig. 6. Transconductance-C circuit implementing membrane dynamics for one neuron with synaptic input from the other three neurons.

yields a membrane current

$$
I_{N a}=\frac{\kappa}{V_{t h}} I_{g_{N a} m^{3} h}\left(V-E_{N a}\right)
$$

where $I_{r e f}$ is the same current reference controlling the amplitude of the gating variables (5) for dimensionless operation. For each of the membrane conductances, one amplifier is connected in parallel as shown in Fig. 6. A capacitance $C_{m e m}=C$ on the membrane node realizes the membrane dynamics (1). To enforce a consistent temporal scale of the dynamics across membrane and gating variables, the currents implementing the opening and closing rates and membrane conductances are globally scaled with a current $I_{\tau}$ that drives the multiplying DACs:

$$
\begin{aligned}
I_{\alpha} & =\alpha I_{\tau} \\
I_{\beta} & =\beta I_{\tau} \\
I_{g} & =g I_{\tau}
\end{aligned}
$$

and thus uniformly controls the time base of all dynamic variables with a global temporal scale parameter $\tau=\frac{C V_{T}}{I_{\tau}}$.

\section{EXPERIMENTAL RESULTS}

The NeuroDyn chip was fabricated in a AMI $0.5 \mu \mathrm{m}$ process and is shown in Fig. 1(b). The DACs used to store the parameter data take up nearly 60 percent of the chip area. The chip measures $3 \mathrm{~mm} \times 3 \mathrm{~mm}$, and draws $0.39 \mathrm{~mA}$ from a $3.3 \mathrm{~V}$ supply.

\section{A. Programmable Channel Kinetics}

We programmed the gate opening and closing variables for one neuron implementing the $\mathrm{HH}$ model. We performed linear regression to determine the parameters of the spline model based on chip characteristics. With 10-bit programming for each of the 7 spline amplitude levels in the regression functions, an adequate fit was obtained as shown in Fig. 7.

\section{B. Neuron Spiking Dynamics}

We observed the dynamics of the membrane and gating variables for one neuron programmed to implement the $\mathrm{HH}$ model. We also demonstrated temporal control through the 

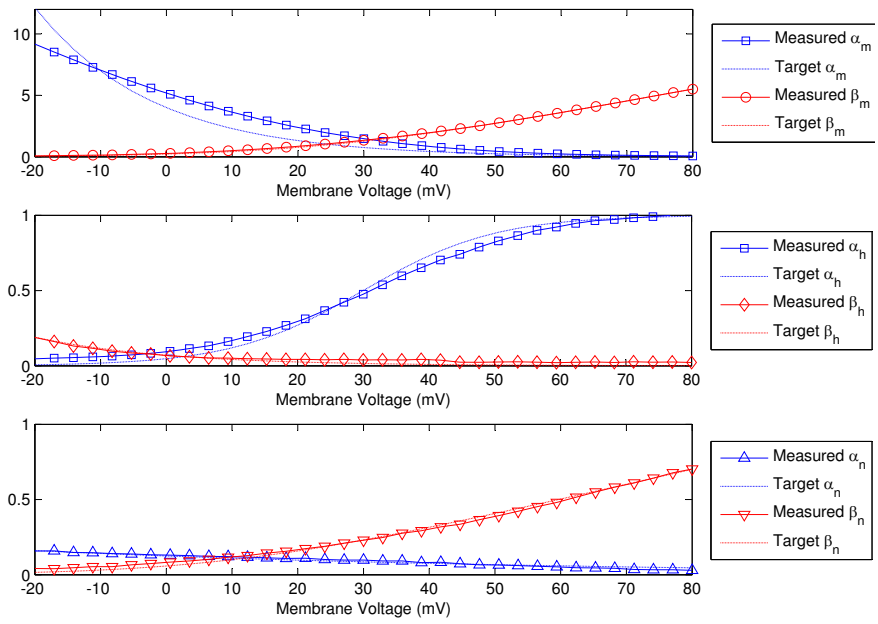

Fig. 7. Target and measured channel opening and closing rates $\alpha$ and $\beta$ for gating variables $n, m$, and $h$ of a $\mathrm{HH}$ neuron, obtained by fitting of the on-chip programmed parameters.

variation of the global temporal scale parameter set by current $I_{\tau}$. As shown in Fig. 8, a two-fold variation of $I_{\tau}$ scales the time axis of the waveforms observed on the oscilloscope by a factor of two. The amplitude scaling in the gating parameters reflects their scaling by $I_{\tau}$ consistent with (8), (9), and (10).

\section{CONClusion}

We present a fully connected biophysical network of neurons and synapses with digitally programmable analog membrane dynamics and channel kinetics. Future work includes further testing of various network parameters and interfacing with a computer for experimental testing of network dynamics. All configurable parameters in the implemented model have a biophysical origin, thus supporting direct interpretation of the results of adapting/tuning the parameters in terms of neurobiology and effects of aging on neural dynamics.

\section{ACKNOWLEDGMENT}

The authors would like to thank Stephen Deiss and Mike Chi for help with the chip design and experimental setup. This work was funded by NIH R01AG029681. The design of the chip originated in a class project at UCSD, and the chip was fabricated through the MOSIS Educational Program. The authors would also like to acknowledge the 2008 NSF Workshop on Neuromorphic Cognition Engineering in Telluride, Colorado.

\section{REFERENCES}

[1] Mead, C.A., Analog VLSI and Neural Systems, Addison-Wesley. 1989.

[2] Hogdkin, A.L.; Huxley, A.F. "A Quantitative Description of Membrane Current and Its Application to Conduction and Excitation in Nerve," J. Physiol., vol. 117, pp. 500-544. 1952

[3] Andreou, A.; Boahen, K. "Translinear Circuits in Subthreshold MOS," Analog Integrated Circuits and Signal Processing, vol. 9, pp. 141-166. 1996

[4] Simoni, Mario F.; Cymbalyuk, Gennady S.; Sorensen, Michael E.; Calabrese, Ronald L.; DeWeerth, Stephen P. "A Multiconductance Silicon Neuron With Biologically Matched Dynamics," IEEE Transactions on Biomedical Engineering, vol. 51, no. 2. 2004
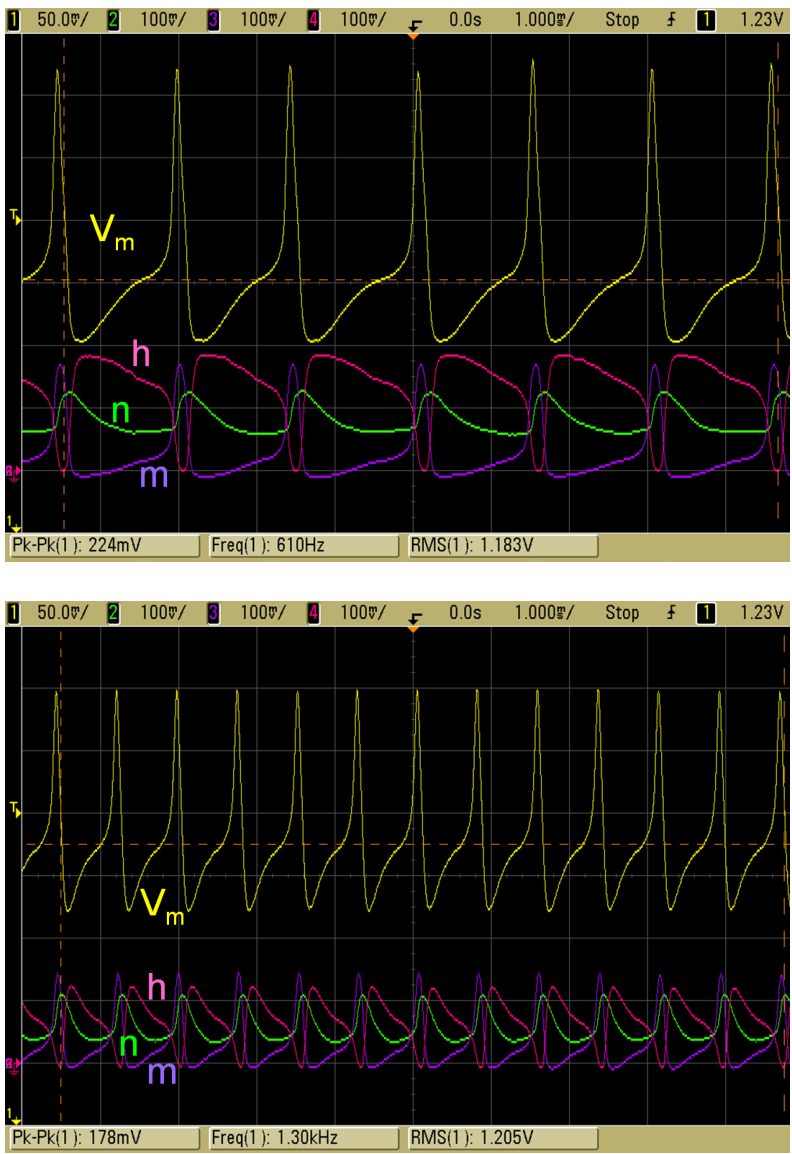

Fig. 8. Measured dynamics of membrane voltage $V_{m}$ and gating variables $n, m$, and $h$ for a single HH neuron. (a) and (b) show the effect of setting the global temporal scale parameter $I_{\tau}$, uniformly speeding or slowing the dynamics across all variables.

[5] Farquhar, E.; Hasler, P. "A Bio-Physically Inspired Silicon Neuron," IEEE Transactions on Circuits and Systems - 1: Regular Papers, vol. 52, no. 3. 2005

[6] Le Masson, S.; Laflaquiere, A.; Bal, T.; Le Masson, G. "Analog Circuits for Modeling Biological Neural Networks: Design and Application," IEEE Transactions on Biomedical Engineering, vol. 46, no. 6. 1999

[7] Georgiou, J.; Drakakis, E.M.; Toumazou, C.; Premanoj, P. ”An Analogue Micropower Log-Domain Silicon Circuit for the Hodgkin and Huxley Nerve Axon," Proc. IEEE ISCAS 1999, vol.2, pp.286-289. 1999

[8] Saighi, S.; Bornat, Y.; Tomas, J.; Renaud, S. "Neuromimetric ICs and System for Parameters Extraction in Biological Neuron Models," Proc. IEEE ISCAS 2006, pp. 4207-4211. 2006

[9] Destexhe A., Z.F. Mainen and T.J. Sejnowski, "Synthesis of Models for Excitable Membranes, Synaptic Transmission and Neuromodulation Using a Common Kinetic Formalism," J. Comp. Neuroscience, vol. 1, pp. 195-230. 1994.

[10] Hynna, K.; Boahen, K. "Thermodynamically Equivalent Silicon Models of Voltage-Dependent Ion Channels," Neural Computation, vol. 19, pp. 327-350. 2007

[11] Furth, P.M.; Andreou, A.G. "Linearised Differential Transconductors in Subthreshold CMOS," Electronics Letters Online, vol. 31, no. 7. pp. 545546. 1995

[12] Vogelstein, R.J.; Mallik, U.; Vogelstein, J.T.; and Cauwenberghs, G. "Dynamically Reconfigurable Silicon Array of Spiking Neurons With Conductance-Based Synapses," IEEE Trans. Neural Networks, vol. 18(1), pp. 253-265. 2007 